\title{
Novel RF-MEMS capacitive switching structures
}

\author{
X. Rottenberg, H. Jansen*, P. Fiorini, W. De Raedt and H. A. C. Tilmans. \\ IMEC v.z.w., Division MCP, Kapeldreef 75, B3001 Leuven, Belgium, xrottenb@imec.be \\ * Twente Univ., MESA+ Res. Ins., Elec. Eng. Dpt., PO Box 217, 7500 AE Enschede, Netherlands
}

\begin{abstract}
This paper reports on novel RF-MEMS capacitive switching devices implementing an electrically floating metal layer covering the dielectric to ensure intimate contact with the bridge in the down state. This results in an optimal switch down capacitance and allows optimisation of the down/up capacitance ratio all that are not possible with standard capacitive switches. Simulation data and measurement results are presented clearly indicating the improved performance characteristics compared to standard RF-MEMS capacitive switches, in the frequency range from $1 \mathrm{to} 30 \mathrm{GHz}$. Down/up capacitance ratios higher than 450 have been measured, an improvement of a factor 34 over standard designs with equal size and using the same materials.
\end{abstract}

\section{INTRODUCTION}

RF-MEMS switches offer great potential benefits over GaAs MMICs and PIN diode switches for application in wireless communication systems [1-3]. Prototype RFMEMS switches display low loss $(<0.4 \mathrm{~dB})$, good isolation $(>20 \mathrm{~dB})$, extremely low standby power consumption, excellent linearity (IP3 $>66 \mathrm{dBm}$ ), compactness and high levels of integration [2-6]. A typical built-up of a RF-MEMS capacitive switch in a shunt configuration implemented on a CPW (CoPlanar Waveguide) line is shown in Figure 1 [4-6]. The switch consists of a suspended movable metal bridge, which is mechanically anchored and electrically connected to the ground of the CPW.

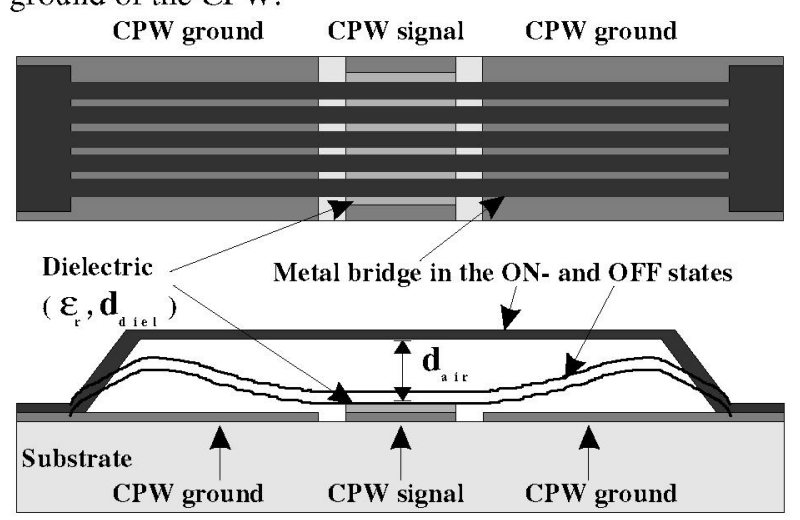

Figure 1: Standard RF-MEMS capacitive shunt switch

To first order, the switch can be modeled as a capacitor between the metal bridge and the signal line. In the RFON state the bridge is up, hence the switch capacitance is small, hardly affecting the impedance of the line. By applying a DC bias (superimposed on the RF signal) the bridge is pulled down onto the dielectric, the switch capacitance becomes high and the switch is OFF or in the isolation state. An important figure of merit quantifying the RF performance is the down/up capacitance ratio,
$\mathrm{C}_{\text {down }} / \mathrm{C}_{\text {up }}$, which must be as high as possible. This ratio can be approximated by

$$
C_{\text {down }} / C_{\text {up }} \approx \frac{\varepsilon_{0} \varepsilon_{r}^{A_{\text {overlap }}} / d_{\text {diel }}}{\varepsilon_{0} A_{\text {overlap }}}=\varepsilon_{r} \frac{d_{\text {air }}}{d_{\text {diel }}}
$$

where $d_{\text {air }}$ and $d_{\text {diel }}$ are the thickness of the air gap and the dielectric, respectively, $\varepsilon_{\mathrm{r}}$ is the dielectric constant of the dielectric and $\mathrm{A}_{\text {overlap }}$ is the overlap area of the bridge and the signal line. For a given technology, as $\mathrm{A}_{\text {overlap }}$ cancels in (1), the isolation imposes the insertion loss and vice versa. The design freedom is thus heavily constrained.

A second problem encountered in capacitive switches of the type shown in Figure 1 is the degradation of the effective down capacitance as a result of surface roughness preventing intimate contact between beam and dielectric [5]. The commonly pursued solutions to attain a large down capacitance are aimed at keeping the roughness of the bridge and of the dielectric layer very low, e.g., $<5 \mathrm{~nm}$, and to keep the surface free from residues [4-6]. Muldavin et al. [5] and Yao et al. [6] introduced thin bottom metals in an attempt to reduce the roughness. In particular, in [4], a thin refractory metal layer (e.g., W) was used. All these measures however lead to a high series resistance hence to an increased insertion loss for a shunt switch. Obviously, in a standard design as the one shown in Figure 1, a difficult compromise must be made. The methods for improving the isolation directly lead to a deterioration of the insertion loss.

In this paper, a novel RF-MEMS switching structure is introduced in which the isolation is optimised without compromising the insertion loss. In addition a new degree of freedom is introduced to equation (1) that allows us to improve on the standard capacitance ratio by an order of magnitude. 


\section{SWITCH DESIGN}

The basic design is shown in Figure 2. A SEM picture of a fabricated device is shown in Figure 3. Key in the design is the use of an electrically floating metal layer covering the dielectric.

The concept of using the floating metal is to ensure that an optimal down capacitance can be achieved without having to resort to very smooth surfaces. A few ohmic contact points between the bridge and the floating metal suffice to attain the optimal down-capacitance given by $C_{\text {down }}=\varepsilon_{0} \cdot \varepsilon_{r} \cdot A_{\text {float }} / d_{\text {diel }}$, where $A_{\text {float }}$ is the area of the floating metal. It further allows the use of a thick, e.g., same thickness as usual CPW lines, highly conductive, and thus low-loss bottom metal layer. The only requirement is that the bridge-to-floating metal contact impedance (combination of contact resistance and capacitance due to a native oxide layer) is sufficiently low so as not to limit the best attainable isolation.

As can be seen in Figure 2 and Figure $3, \mathrm{C}_{\text {down }}$ is defined by the area of the floating top metal $\left(\mathrm{A}_{\text {float }}\right.$ ) whereas $\mathrm{C}_{\mathrm{up}}$ is still determined by the area $A_{\text {overlap }}$, the overlap region between the bridge and the signal line which is independent of $\Lambda_{\text {float }}$. This allows to further optimise the capacitance ratio. The up-capacitance can be lowered, by choosing a bridge more narrow than the floating top metal, without affecting the down capacitance. We can thus decouple the two switching states and introduce a geometrical factor that "boosts" the capacitance ratio to:

$$
C_{\text {down }} / C_{\text {up }} \approx \frac{\varepsilon_{0} \varepsilon_{r}^{A_{\text {float }} / d_{\text {diel }}}}{\varepsilon_{0} A_{\text {overlap }} / d_{\text {air }}}=\varepsilon_{r} \frac{d_{\text {air }}}{d_{\text {diel }}} \frac{A_{\text {float }}}{A_{\text {overlap }}}
$$

The introduction of the floating top metal requires a revision of the actuation scheme of the standard switch of Figure 1. Just covering the dielectric with a floating metal would result in an unstable device. If, in this case, a bias is applied, the bridge pulls in but releases as soon as it touches the floating top metal. Upon contact, the floating top metal and the bridge have the same potential. In other words, the electrostatic attractive force vanishes.

The structure shown in Figure 2 and Figure 3 can be actuated in two ways. One way is via separate actuation electrodes located in area 1 adjacent to the signal line as shown. The other way uses the small areas adjacent to the floating top metal and part of the switch capacitance, indicated as area 2 . Using actuation area 1 , the capacitive contact can be replaced by an ohmic contact as recently demonstrated by Tan et al [7]. In using actuation area 2 a capacitive switch results with the exception that a floating top metal is used.
In a final design the actuation area 1 will no longer be used, thus obtaining a very compact capacitive shunt switching device.

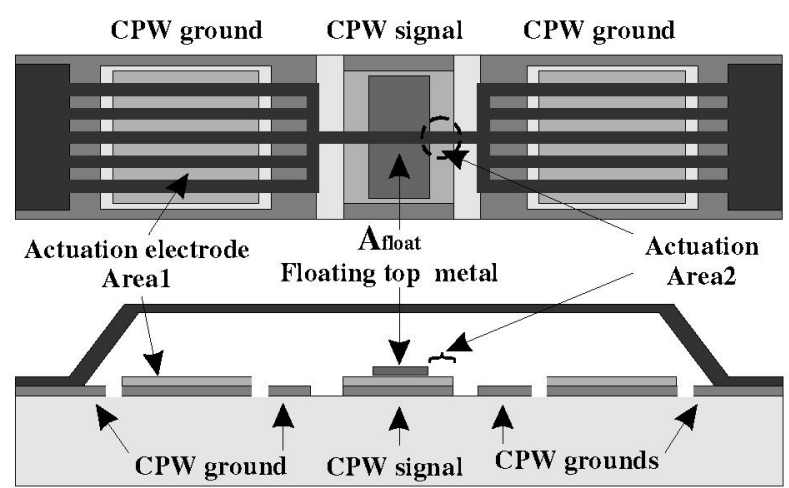

Figure 2: RF MEMS boosted switching structure.

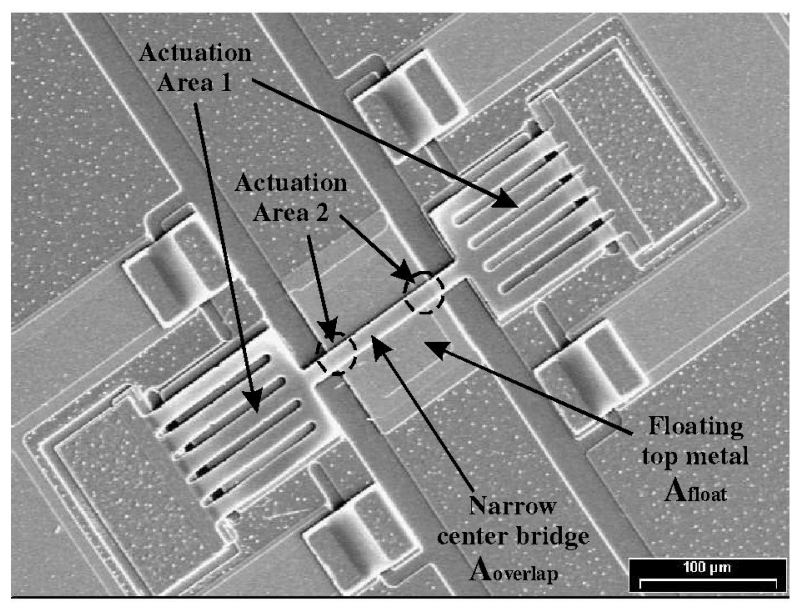

Figure 3: SEM of the boosted RF MEMS switching device.

\section{PROCESS SEQUENCE}

The basic steps of the processing can be described as follows. A stack of $1 \mu \mathrm{m} \mathrm{Al}, 200 \mathrm{~nm} \mathrm{Ta} \mathrm{O}_{5}$ and $100 \mathrm{~nm}$ $\mathrm{Al}$ is deposited on a $700 \mu \mathrm{m}$ thick $\mathrm{AF} 45$ glass substrate. A first mask is used to etch both the top $\mathrm{Al}$ and the dielectric to define the area where the bottom $\mathrm{Al}$ will be protected. The second mask is used to define the floating top metal plate. The 50/100/50 $\mu \mathrm{m}$ CPW lines and the top $\mathrm{Al}$ for the actuation area 1 are defined with mask \#3.

Next a $3 \mu \mathrm{m}$ thick polymer sacrificial layer is spun and patterned to define the bridge anchors. A $1 \mu \mathrm{m} \mathrm{Al}$ layer is sputtered and etched defining the bridge. The bridges are released in a final sacrificial layer plasma etch.

\section{SIMULATIONS AND MEASUREMENTS}

The $200 \mu \mathrm{m}$ long switch areas are fed through $200 \mu \mathrm{m}$ long line sections. 
Figure 4 and Figure 5 show the simulated S-parameters $\left(\mathbf{S}_{21}\right)$ using HFSS (High Frequency Structure Simulator) for the various switches in the ON-state and the OFFstate, respectively. In Figure 4, a simulation of a $600 \mu \mathrm{m}$ CPW line is included for reference purposes.

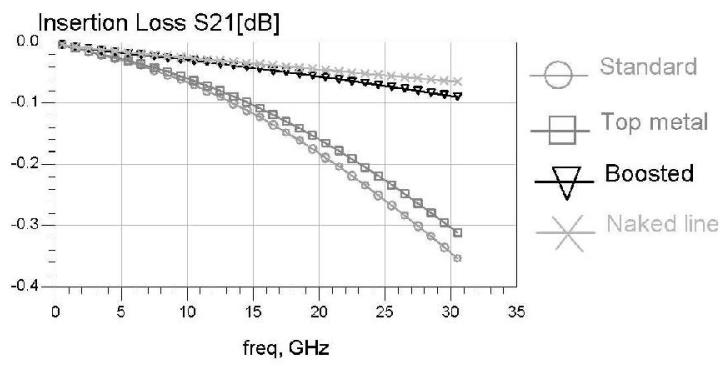

Figure 4:HFSS Insertion Loss simulations of standard switch, switch with floating top metal, boosted switch and naked line

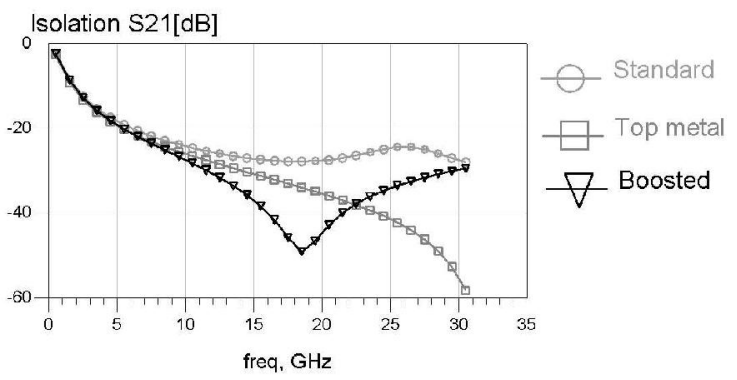

Figure 5: HFSS Isolation simulations of standard switch, switch with floating top metal and boosted switch

The simulations indicate that the same insertion loss is obtained for the standard and the floating top metal devices, but that the boosted switch brings about the expected improvement in the ON-state (bridge in the upstate). The "naked" CPW line is nearly non-sensitive to the presence of a narrow bridge. The loss of the boosted device is only slightly higher than the loss of the naked line $(0.09 \mathrm{~dB}$ vs. $0.07 \mathrm{~dB}$ at $30 \mathrm{GHz}$ for the line).

In the simulations of Figure 5 (switch in down state) a perfect contact between the bridge and the dielectric is assumed. The dip in the curve is well-known and is due to the LC resonance [5]. It is found, that the isolation for all structures is the same at low frequencies $(<5 \mathrm{GHz})$. At higher frequencies, the standard switch starts to behave as a number of LC-tanks in parallel, due to the ribbonlike bridge design (see Fig. 1). This broadens the LC resonance dip. The switch with top metal effectively has only a single LC tank giving a single sharp resonance peak. The boosted switch shows similar behaviour but the $\mathrm{LC}$ resonance occurs at higher frequency due to the higher inductance of the narrow center bridge. Note that tuning of the LC resonance frequency by shaping the bridge offers the opportunity to greatly improve the isolation in a determined bandwidth. This is of interest for the low frequency application where the shunt switch, by nature, has poor performances.

The insertion loss and the isolation of the various configurations have been measured and are shown in Figure 6 and Figure 7, respectively. In Figure 7 a fourth curve, labelled as "dummy", has been added. The dummy represents a bridge permanently fixed during fabrication in the down-state. It is free from any contact problems and represents the optimum bridge shape when pulled down. The dummy is used as the best possible reference to evaluate and compare the actual switches.

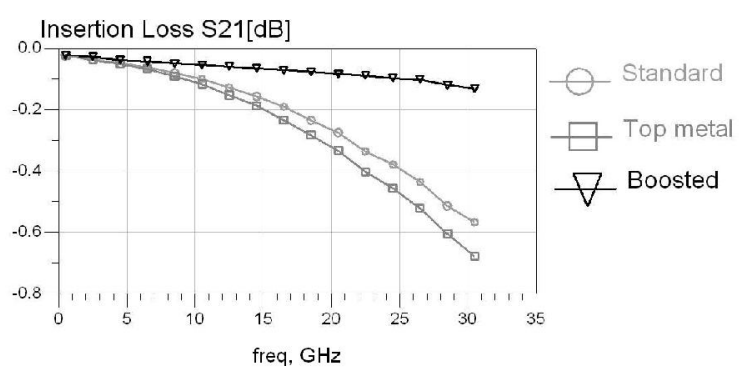

Figure 6: Insertion loss measurements

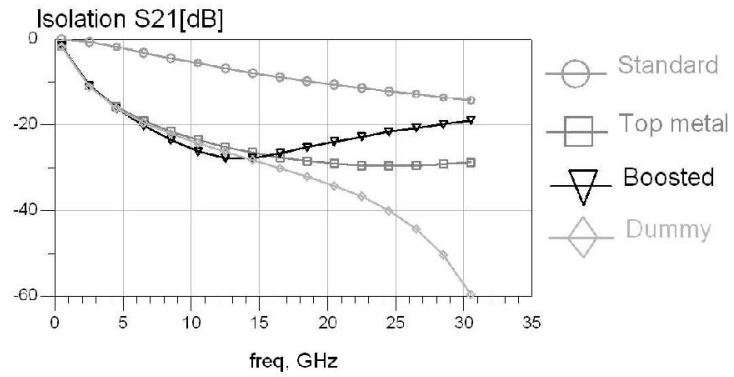

Figure 7: Isolation measurements

The insertion loss measurements show a good agreement with the simulations although different by a factor 2 .

The source of this discrepancy has not been explained yet. As predicted by the simulations (Figure 4), adding a floating top metal only slightly modifies the behaviour of the switch, increasing the insertion loss with $0.1 \mathrm{~dB}$ at $30 \mathrm{GHz}$ as compared to the standard switch. The boosted switching device shows a much lower loss than the other two device structures. This is explained by the much smaller up-state capacitance of the boosted device. The loss of the boosted switch is now limited by the losses of the $1 \mu \mathrm{m}$ thick $\mathrm{Al}$ CPW line.

The isolation measurement (Figure 7) on the standard switch shows the typical behaviour of a capacitive switch with poor bridge-dielectric contact. It clearly differs from the simulations. The isolation measurements on the other structures shows good agreement with the simulations. They present almost the same low frequency behaviour, the only differences appearing in the $\mathrm{LC}$ resonance 
characteristics. It seems that the dummy structure goes to a sharp LC resonance around $30 \mathrm{GHz}$, whereas a clear $\mathrm{LC}$ resonance is not observed for the other switching devices. The higher series resistance R (see Figure 8) leading to a higher damping and thus a broader dip explains this observation.

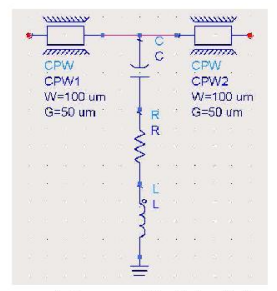

Figure 8: Model
The switches are modelled with the circuit shown in Figure 8. The measurements were fitted on this model using $\mathrm{R}, \mathrm{L}$ and $\mathrm{C}$ as the fitting parameters, with a fixed model for the CPW line sections. The fitting parameters are summarized in Table 1.

Table 1: Measurement extracted fitting parameters.

\begin{tabular}{|l|c|c|c|c|c|}
\hline & $\begin{array}{c}\mathrm{C}_{\mathrm{up}} \\
{[\mathrm{fF}]}\end{array}$ & $\begin{array}{c}\mathrm{C}_{\text {down }} \\
{[\mathrm{pF}]}\end{array}$ & $\begin{array}{c}\mathrm{L}_{\text {down }} \\
{[\mathrm{pH}]}\end{array}$ & $\begin{array}{c}\mathrm{R}_{\text {down }} \\
{[\Omega]}\end{array}$ & $\frac{C_{\text {down }}}{C_{u p}}$ \\
\hline Standard & 68 & 0.90 & $(2)$ & $(0.22)$ & 13 \\
\hline Top metal & 78 & 7.98 & 10 & 1.12 & 102 \\
\hline Boosted & 18 & 7.98 & 18 & 1.33 & 459 \\
\hline Dummy & - & 9.12 & 10 & 0.15 & - \\
\hline
\end{tabular}

The up-capacitance of the standard switch and the switch with top metal do not differ significantly. The boosted switch (with a 5 times narrower center bridge), displays a 4 times smaller up-capacitance. The down capacitance of the top-metallized and boosted switches is the same, and is a factor 9 better than the down-capacitance of the standard switch. The difference between the $7.98 \mathrm{pF}$ measured on the movable structures and the $9.12 \mathrm{pF}$ measured on the dummy structures is due to the difference in contact areas (equal to the "pulling area 2" explained in Figure 2 and Figure 3).

For frequencies (much) smaller than the LC resonance, the capacitance dominates the RLC impedance. The small $\mathrm{C}_{\text {down }}$ observed for the standard switch shifts the $\mathrm{LC}$ resonance to very high frequencies, out of our measurement range. The extracted $R_{\text {down }}$ and $L_{\text {down }}$ for the standard switch are therefore only poor estimates. For the other configurations, the extracted L- and R-values are more reliable. The inductance for the switch with top metal and the dummy switch is the same, while the boosted switch shows a much higher inductance.

A resistance close to $1 \Omega$ has been extracted for the movable devices using top metal (including the boosted switch), while the dummy shows a much smaller resistance of $0.2 \Omega$. This can be explained by the thicker top metal of the dummy, which is $1 \mu \mathrm{m} \mathrm{Al}$ vs. $0.1 \mu \mathrm{m}$ thick $\mathrm{Al}$ for the other configurations.
Most importantly the calculated capacitance ratio improves from 13 for the standard switch to above 400 for the boosted device. Truly considered an impressive improvement for the boosted device.

\section{CONCLUSIONS}

Novel RF-MEMS capacitive switching devices are presented. A feature of the device is that the insertion and the isolation can be independently optimised, impossible with standard switch designs. Due to this feature, the devices offer a high down-capacitance and a down-to-up capacitance ratio as high as 450 , an improvement with a factor 34 over standard designs. The measured insertion loss is below $0.06 \mathrm{~dB}$ in the range $1-15 \mathrm{GHz}$ (and includes the losses of the $600 \mu \mathrm{m}$ long lines estimated to be around $0.04 \mathrm{~dB}$ ). The isolation higher than $25 \mathrm{~dB}$ at $15 \mathrm{GHz}$ has been measured. It is expected that further improvements are still possible based on this concept.

\section{ACKNOWLEDGEMENT}

This project was supported by ESA-ESTEC under contract $14627 / 00 / \mathrm{NL} / \mathrm{KW}$. The authors in particular would like to thank François Deborgies and Laurent Marchand from ESTEC for the many fruitful discussions. Further acknowledgements go to all the members of the HDIP and MEMS groups of IMEC and to the L.E.T.

\section{REFERENCES}

(1) C. T.-G. Nguyen, et al., "Micromachined devices for wireless communications", Proc. of the IEEE, vol. 86(8), 1998, pp. 1756-1768

(2) J. J. Yao, "RF MEMS from a device perspective", J. Micromech. Microeng., vol.10(4), December 2000, pp. R9-R38.

(3) G. M. Rebeiz and J. B. Muldavin, "RF MEMS switches and switch circuits", IEEE Microwave magazine, Dec. 2001, pp. 59-71.

(4) Z. Jamie Yao, et al., "Micromachined low-loss microwave switches", IEEE J. of MEMS, vol. 8(2), 1999, pp. 129-134.

(5) J. B. Muldavin and G. M. Rebeiz, "High-isolation CPW MEMS shunt switches-Part 1: Modeling", IEEE Trans. Microwave Theory and Techniques, vol. 48(6), 2000, pp. 1045-1052.

(6) H. A. C. Tilmans, et al., "Wafer-level packaged RF-MEMS switches fabricated in a CMOS fab", proc. IEDM 2001, Washington, DC, December 3-5, 2001, pp. 921-924.

(7) G.-L. Tan and G. M. Rebeiz, "DC-26 GHz MEMS seriesshunt absorptive switches", proc. IEEE MTT-S, Phoenix, AZ, 20-25 May, 2001, pp. 325-328, 2001. 